\title{
N. Paucivorans Infection Presenting as a Brain Abscess
}

\author{
Shah-Naz Hayat Khan, Stephen E. Sanche, Christopher A. Robinson, Farhad Pirouzmand
}

Can. J. Neurol. Sci. 2006; 33: 426-427

We report an intracerebral abscess caused by a recently identified nocardial species, in an immunocompetent individual without extraneural involvement.

A 63-year-old non-smoking Caucasian male with an unremarkable history of hypertension, presented with a 2-week history of personality changes including apathy, some difficulty walking, a tendency to veer to the left and a possible seizure. Preliminary assessment, including laboratory investigations were unremarkable. Computed tomography (CT) of his brain revealed a right frontal lobe multi-loculated, ring-enhancing lesion with vasogenic edema and associated mass effect (Figure 1). He was then referred to our institution.

We found the patient afebrile, with no other focal neurological deficits. Repeat investigations including white blood cell count and chest $\mathrm{x}$-ray, were normal. With a presumptive diagnosis of brain tumor, the patient was started on oral steroids and anti-seizure medication. A right frontal craniotomy was performed. Using bipolar cautery, a small corticectomy was made in the abnormal hyperemic brain surface overlying the lesion. An odorless, yellowish, creamy, pus-like discharge emanated from just underneath the cortex. Further dissection revealed a thick walled and multi-loculated cavity. Specimens were collected and the site was copiously irrigated to remove the pus-like material. The lateral ventricle was carefully avoided. Broad-spectrum coverage with ceftriaxone and metronidazole was commenced. Tissue histopathology returned consistent with Nocardia. Therefore, the antibiotics were changed to intravenous (i.v.) trimethoprim/sulfamethoxazole (TMP/SMX) and metronidazole. Metronidazole was discontinued when the anaerobic cultures returned negative.

Tissue and fluid specimens were cultured on blood and chocolate agars. Gram-positive, non-hemolytic, catalase positive, weakly acid-fast, filamentous, branching bacilli were observed within 72 hours. The organism was presumptively identified as a Nocardia species. The Canadian Science Centre for Human and Animal Health confirmed it as N. paucivorans, based on 16S rDNA gene sequencing and conventional biochemical tests.

Post-operatively, the patient made a rapid recovery and remained without focal deficits. The $\mathrm{CT}$ scan demonstrated a residual cavity behind the area of resection. Further investigations did not reveal any evidence of immunocompromise, or of nocardiosis elsewhere in the body. After a week in hospital, the patient was discharged home on i.v. TMP/SMX.

Trimethoprim/sulfamethoxazole was changed to oral route when a CT, done three weeks postoperatively, showed marked attenuation of the residual abscess. Complete resolution was

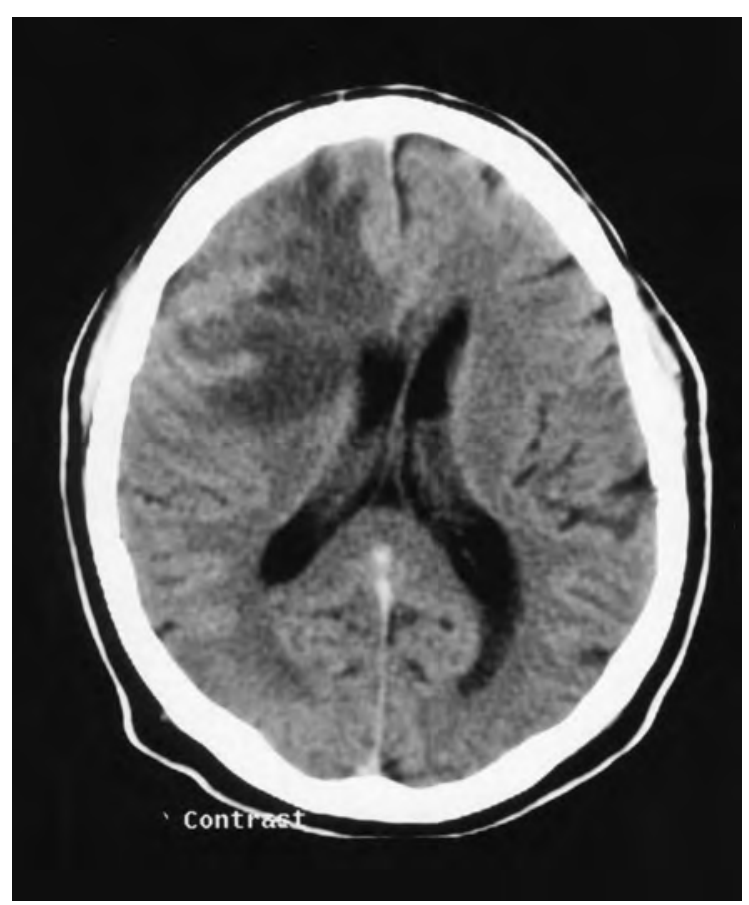

Figure 1: Computed tomography scan with contrast. The preoperative scan shows an enhancing, multi-loculated lesion in the right frontal lobe.

documented on the CT performed three months postoperatively (Figure 2). Antibiotics were discontinued. When last evaluated nine months after discharge, the patient continued to remain well, with no recurrent abscess on CT.

Nocardiosis is primarily a pulmonary disease. It causes up to $2 \%$ of cerebral abscesses, mostly in the immuno-

From the Division of Neurosurgery, Department of Surgery (SHK), Division of Infectious Diseases, Department of Medicine (SES), Department of Pathology (SES, CAR); Saskatoon Health Region (SES, CAR), Saskatoon, SK, Canada; Division of Neurosurgery (FP), Sunnybrook and Women's College Health Science Centre, University of Toronto, Toronto, ON, Canada.

ReCEIVEd August 12, 2005. ACCEPTED IN FINAL FORM June 27, 2006. Reprint requests to: S.H. Khan, Department of Neurosurgery,University of Cincinnati Medical Center, PO Box 670515, Cincinnati, Ohio, 45267-0515, U.S.A. 


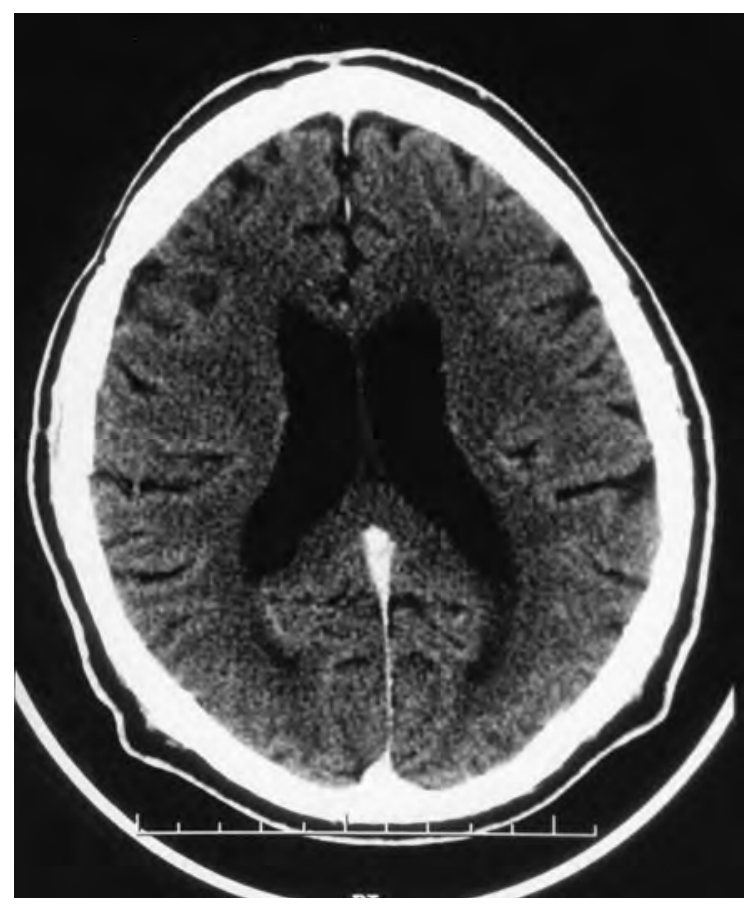

Figure 2: Computed tomography scan with contrast. Complete resolution of the abscess following 3 months of treatment.

compromised. ${ }^{1,2,3}$ Increasingly, it is being recognized in immunocompetent individuals with cerebral abscesses. ${ }^{4,5,7}$ Primary cerebral abscesses without evidence of pulmonary infection are unusual. ${ }^{3}$ The immune status and the timeliness of treatment influence outcome, so that the immunocompetent patient receiving early treatment intervention will do better. ${ }^{2,3,5,6}$

To date, 73 Nocardia species have been identified, including 42 additions since $2000 .{ }^{9}$ Only a few species are neuroinvasive, with $N$. asteroides being the most frequent. ${ }^{2,5,6}$ There are three previous reports of $N$. paucivorans infection. ${ }^{10,12}$ Two patients were immunocompromised and had intracranial abscesses. The third had chronic pulmonary disease and $N$. paucivorans was isolated from sputum. In our case, the patient was immunocompetent and without any chronic debilitating disorders.

Based on history and radiological findings, we made a presumptive diagnosis of a neoplastic process, with abscess considered less likely. A slow, afebrile presentation with radiological imaging consistent with brain tumor, has been reported in cerebral nocardiosis. ${ }^{5,7}$ In previous reports, diagnosis of a neoplastic process was revised following operative findings and pathology results. ${ }^{2-5,13}$ Preoperative magnetic resonance imaging or MR spectroscopy most probably would have made the distinction between an abscess and a tumor. However, that information would not have altered the intent of early surgical intervention.

Compared with poor outcomes in other cases, ${ }^{3,7}$ our plan of early surgery and prolonged antibiotics administration, led to a complete recovery. Unlike previous reports, ${ }^{7,14}$ we were able to administer steroids for a week post-operatively without any adverse effects. The duration of antibiotic therapy remains empirical. ${ }^{2,3,5,13}$ We used clinical, laboratory and radiological evidence to determine the duration. Antibiotics were administered i.v. for a month and orally for 3 months (total=4 months), effecting a cure. In vitro data supports the efficacy of TMP/SMX in $N$. paucivorans infections. ${ }^{10,11}$

This case highlights nocardial cerebral abscesses in immunocompetent patients, where the residual nocardial abscess can be successfully treated with antibiotics. Further studies are needed to better understand the pathogenesis, improve management and establish preventive measures.

\section{ACKNOWLEDGEMENTS}

The authors thank Dr. Katherine Bernard and her associates at the Canadian Science Centre for Human and Animal Health for their kind assistance.

Dr S.H. Khan was a resident sponsored by Saskatoon Health Region.

\section{REFERENCES}

1. Palmer DL, Harvey RL. Wheeler J.K. Diagnostic and therapeutic considerations in Nocardia asteroides infection. Medicine. 1974;63:391-401.

2. Mamelak AN, Obana WG, Flaherty JF, Rosenblum ML. Nocardial brain abscess: treatment strategies and factors influencing outcome. Neurosurgery. 1994;35:622-31.

3. Byrne E, Brophy BP, Perrett LV. Nocardia cerebral abscess: new concepts in diagnosis, management, and prognosis. J Neurol Neurosurg Psychiatry. 1979;42:1038-45.

4. Mogilnor A, Jallo GI, Zagzag D, Kelly P. Nocardia abscess of the choroid plexus: clinical and pathological case report. Neurosurgery. 1998;43:949-52.

5. Fleetwood IG, Embil JM, Ross IB. Nocardia asteroides cerebral abscess in immunocompetent hosts: report of three cases and review of surgical recommendations. Surg Neurol 2000;53:60510.

6. Valerzo J, Cohen JE, Valerzo L, Spektor S, Shoshan Y, Rosenthal G, et al. Nocardial cerebral abscess: report of three cases and review of the current neurosurgical management. Neurol Res. 2003;25:27-30.

7. Roquer J, Pou A, Herraiz J, Campodarve I, Sequeira T, Vilató J, et al. Primary cerebral abscess due to Nocardia presenting as 'Ghost Tumor'. Eur Neurol. 1990;30:254-7.

8. Beaman BL, Beaman L. Nocardia species: host-parasite relationships. Clin Microbiol Rev. 1994;7:213-64.

9. Euzéby JP. List of bacterial names with standing in Nomenclature Genus Nocardia. Int J Syst Bacteriol. http://www.bacterio. cict.fr/n/nocardia.html.

10. Eisenblätter M, Disko U, Stoltenburg-Didinger G, Scherübl H, Schaal KP, Roth A, et al. Isolation of Nocardia Paucivorans from the cerebrospinal fluid of a patient with relapse of cerebral nocardiosis. J Clin Microbiol. 2002;40:3532-4.

11. Wellinghausen N, Pietzcker T, Kern WV, Essig A, Marre R. Expanded spectrum of Nocardia species causing clinical nocardiosis detected by molecular methods. Int $\mathrm{J}$ Med Microbiol. 2002;29:277-82.

12. Yassin AF, Rainey FA, Burghardt J, Brzezinkz H, Mauch M, Schaal KP. Nocardia Paucivorans sp. nov. Int J Syst Evol Microbiol. 2000;50 Pt 2:803-9.

13. Smith PW, Steinkraus GE, Henricks BW, Madson EC. CNS nocardiosis response to sulfamethoxazole-trimethoprim. Arch Neurol. 1980;37:729-30.

14. Goodman JS, Koenig MG. Nocardia infections in a general hospital. Ann NY Acad Sci. 1970;174:552-67. 